\section{A perspective on advanced manufacturing and AMP2.0}

\author{
www.manufacturing.gov/nnmi.html
}

The world's first three-dimensional printed car, designed with open-source code and assembled in 44 hours ... an ultra-lightweight trailer, made from carbon fiber to enable 4000 pounds of additional freight ... National Manufacturing Day, a Presidential declaration to inspire young people in the United States to pursue careers in manufacturing and engineering.

$\mathrm{T}_{\mathrm{s}}^{\mathrm{h}}$ hese recent examples illustrate the growing momentum of advanced manufacturing, defined as the innovative technologies and related processes that can grow productivity, speed product development, and customize products to offset higher wages and cost." In the race to lead the global marketplace, competitive advantages enabled by advanced manufacturing technologies are crucial for success. Furthermore, investments in manufacturing create significant additional economic activity - manufacturing has the largest multiplier effect of any other major economic activity, generating USD \$1.35 in additional economic activity for every $\$ 1$ spent, according to the US Department of Commerce Bureau of Economic Analysis.

Recognizing the vast potential of investments in advanced manufacturing, the President's Council of Advisors on Science and Technology (PCAST) launched a series of national-level discussions and actions in 2011, known as the Advanced Manufacturing Partnership (AMP), to ensure a new era of manufacturing in the United States. AMP brought together industry, universities, government, and other stakeholders to identify emerging technologies with the potential to create high-quality domestic manufacturing jobs and enhance US global competitiveness.

The AMP Steering Committee identified three pillars to ensure an ecosystem for advanced manufacturing leadership: enabling innovation, securing the talent pipeline, and improving the business climate. To enable innovation, AMP recommended public-private partnerships, including the National Network for Manufacturing Innovation (NNMI), to advance high-impact technologies and models for collaboration. The federal government responded by launching five manufacturing institutes by August 2014, with more institutes on the way.

The second phase of AMP (AMP2.0), which began in September 2013, charged PCAST to build on the first set of recommendations in 2012 with further specific, targeted, and actionable recommendations to improve and sustain US manufacturing innovation. To answer this charge, AMP2. 0 convened 43 college and university faculty and administrators and 51 industry leaders and employees, as well as labor group representatives and independent experts for working teams, in addition to contributions from countless participants at regional meetings, roundtables, and other forums.

On October 27, 2014, AMP2.0 released its report, "Accelerating U.S. Advanced Manufacturing," to a packed room at the National Academy of Sciences in Washington, DC. Immediately prior to this highly anticipated public event, the AMP2.0 Steering Committee Co-Chair Rafael Reif, President of the Massachusetts Institute of Technology, delivered the recommendations to President Barack Obama.

The AMP2.0 report reflects the significant engagement by the AMP2.0 participants, while also issuing recommendations built upon the three pillars outlined in the 2012 report. The first pillar concentrates on intensifying the administration's focus on advanced manufacturing, calling for a national strategy that prioritizes technologies, a standing university-industry consortium to provide ongoing technical dialogue with federal policymakers, additional public-private manufacturing research and development infrastructure to support early and maturation stages in technology development on either side of the work done at existing institutes, develop standards and processes to enable interoperability of manufacturing technologies, and a governance structure to continue to grow the NNMI. AMP2.0 also piloted a process for developing a national strategy, focused on three emerging technologies of national importance: advanced sensing, controls, and platforms for manufacturing; visualization, informatics, and digital manufacturing; and advanced materials manufacturing.

The second pillar focuses on securing the talent pipeline, and recommends a national campaign to modernize the current image of manufacturing, stackable skill certifications and federally supported training and accreditation programs to enable the next-generation workforce, and the curation of a toolkit to ease the scale-up and replication of talent development opportunities.

The third pillar seeks to improve the business climate, especially for smalland medium-sized manufacturers, by improving information flow about technologies, markets, and supply chains. AMP2.0 also suggests reducing the risk associated with the scale-up of advanced manufacturing, such as by improving access to capital and tax incentives.

Materials science and engineering is integral to advanced manufacturing, and the materials research community may join the discussion generated by the AMP2.0 report in a variety of ways: contribute to relevant federal requests for information and proposal calls, check www.manufacturing.gov for new materials-related announcements, and consider manufacturing scale-up for materials and processes from concept to design to end of life.

Megan Brewster 2013-2014 Materials Research Society/ The Minerals, Metals and Materials Society Congressional Science and Engineering Fellow

*From S. Berger, Making in America: From Innovation to Market (MIT Press, Cambridge, 2013). 


\section{$2015 \mathrm{M}|\mathrm{R}| \mathrm{S}{ }^{\circ}$ FALL MEETING \& EXHIBIT}
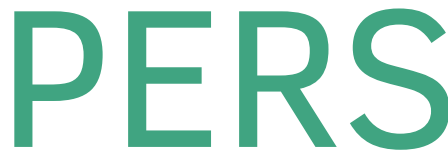

\section{Abstract Submission Opens}

A Engaged Learning of Materials Science and Engineering in the $21^{\text {st }}$ Century

\section{BIOMATERIALS AND SOFT MATERIALS}

B Stretchable and Active Polymers and Composites for Electronics and Medicine

C Tough, Smart and Printable Hydrogel Materials

D Biological and Bioinspired Materials in Photonics and ElectronicsBiology, Chemistry and Physics

E Engineering and Application of Bioinspired Materials

F Biomaterials for Regenerative Engineering

G Plasma Processing and Diagnostics for Life Sciences

H Multifunctionality in Polymer-Based Materials, Gels and Interfaces

I Nanocellulose Materials and Beyond-

Nanoscience, Structures, Devices and Nanomanufacturing

$\mathrm{J}$ Wetting and Soft Electrokinetics

K Materials Science, Technology and Devices for Cancer Modeling, Diagnosis and Treatment

L Nanofunctional Materials, Nanostructures and Nanodevices for Biomedical Applications

\section{NANOMATERIALS AND SYNTHESIS}

M Micro- and Nanoscale Processing of Materials for Biomedical Devices

N Magnetic Nanomaterials for Biomedical and Energy Applications

0 Plasmonic Nanomaterials for Energy Conversion

$P$ Synthesis and Applications of Nanowires and Hybrid 1D-0D/2D/3D Semiconductor Nanostructures

Q Nano Carbon Materials-1D to 3D

R Harsh Environment Sensing-Functional Nanomaterials and Nanocomposites, Materials for Associated Packaging and Electrical Components and Applications

IMECHANICAL BEHAVIOR AND FAILURE OF MATERIALS

$S$ Mechanical Behavior at the Nanoscale

$\mathrm{T}$ Strength and Failure at the Micro- and NanoscaleFrom Fundamentals to Applications

U Microstructure Evolution and Mechanical Properties in Interface-Dominated Metallic Materials

V Gradient and Laminate Materials

W Materials under Extreme Environments (MuEE)

Y Shape Programmable Materials

\section{ELECTRONICS AND PHOTONICS}

Z Molecularly Ordered Organic and Polymer SemiconductorsFundamentals and Devices

AA Organic Semiconductors-Surface, Interface and Bulk Doping

BB Innovative Fabrication and Processing Methods for Organic and Hybrid Electronics

CC Organic BioelectronicsFrom Biosensing Platforms to Implantable Nanodevices

DD Diamond Electronics, Sensors and BiotechnologyFundamentals to Applications

EE Beyond Graphene-2D Materials and Their Applications

FF Integration of Functional Oxides with Semiconductors

GG Emerging Materials and Platforms for Optoelectronics

$\mathrm{HH}$ Optical Metamaterials-

From New Plasmonic Materials to Metasurface Devices

II Phonon Transport, Interactions and Manipulations

in Nanoscale Materials and Devices-Fundamentals and Applications

JJ Multiferroics and Magnetoelectrics

KK Materials and Technology for Non-Volatile Memories

\section{Abstract Submission Deadline June 18, 2015}

\section{ENERGY AND SUSTAINABILITY}

LL Materials and Architectures for Safe and Low-Cost Electrochemical Energy Storage Technologies

MM Advances in Flexible Devices for Energy Conversion and Storage

NN Thin-Film and Nanostructure Solar Cell Materials and Devices for Next-Generation Photovoltaics

00 Nanomaterials-Based Solar Energy Conversion

PP Materials, Interfaces and Solid Electrolytes for High Energy Density Rechargeable Batteries

QQ Catalytic Materials for Energy

RR Wide-Bandgap Materials for Energy EfficiencyPower Electronics and Solid-State Lighting

SS Progress in Thermal Energy Conversion-

Thermoelectric and Thermal Energy Storage Materials and Devices

\section{THEORY, CHARACTERIZATION AND MODELING}

TT Topology in Materials Science-

Biological and Functional Nanomaterials, Metrology and Modeling

UU Frontiers in Scanning Probe Microscopy

VV In Situ Study of Synthesis and Transformation of Materials

WW Modeling and Theory-Driven Design of Soft Materials

XX Architected Materials-Synthesis, Characterization, Modeling and Optimal Design

YY Advanced Atomistic Algorithms in Materials Science

ZZ Material Design and Discovery via Multiscale Computational Materials Science

AAA Big Data and Data Analytics for Materials Science

BBB Liquids and Glassy Soft Matter-Theoretical and Neutron Scattering Studies

CCC Integrating Experiments, Simulations and Machine Learning to Accelerate Materials Innovation

DDD Lighting the Path towards Non-Equilibrium Structure-Property Relationships in Complex Materials

\section{www.mrs.org/fall2015}

The MRS/E-MRS Bilateral Energy Conference will be comprised of the energy-related symposia at the 2015 MRS Fall Meeting.

\section{Meeting Chairs}

T. John Balk University of Kentucky

Ram Devanathan Pacific Northwest National Laboratory George G. Malliaras Ecole Nationale Supérieure des Mines de Saint-Étienne

Larry A. Nagahara National Cancer Institute Luisa Torsi University of Bari "A. Moro"

Don't Miss These Future MRS Meetings! 2016 MRS Spring Meeting \& Exhibit

March 28 - April 1, 2016

Phoenix, Arizona

2016 MRS Fall Meeting \& Exhibit November 27 - December 2, 2016 Boston, Massachusetts

M R S MATERIALS RESEARCH SOCIETY ${ }^{\circledR}$ Advancing materials. Improving the quality of life.

506 Keystone Drive • Warrendale, PA 15086-7573

Tel 724.779 .3003 - Fax 724.779 .8313

info@mrs.org • www.mrs.org 